\title{
Perfil clínico-epidemiológico de pacientes com hanseníase atendidos em hospital universitário no Rio de Janeiro entre 2008 e 2017
}

\author{
Clinical and epidemiological profile of leprosy patients admitted to a university \\ hospital in Rio de Janeiro between 2008 and 2017
}

Vinicius Martins de Menezes ${ }^{1}$, Juliana Chaves Ruiz Guedes ${ }^{2}$, Larissa Starling de Albuquerque Fernandes ${ }^{2}$, Natacha de Mello Haddad², Ricardo Barbosa Lima ${ }^{3}$, Elizabeth Santana Martins ${ }^{4}$, Carlos José Martins ${ }^{5}$

\begin{abstract}
RESUMO
Modelo do estudo: Estudo observacional, longitudinal, retrospectivo e descritivo de uma coorte de pacientes. Objetivo: Descrever o perfil clínico e epidemiológico dos pacientes com diagnóstico de hanseníase, atendidos em um centro de referência no estado do Rio de Janeiro. Metodologia: Foram incluídos todos os pacientes com diagnóstico de hanseníase atendidos no Hospital Universitário Gaffrée e Guinle entre os anos de 2008 a 2017. Os dados foram coletados através da revisão de prontuários e analisados através de estatística descritiva, utilizando-se o software SPSS 22.0 database. Resultados: Um total de 112 pacientes foi incluído no estudo, sendo que 54,5\% eram do sexo masculino. A média de idade foi de 49,9 anos, $62,4 \%$ eram moradores do município do Rio de Janeiro e 69,9\% apresentaram formas clínicas multibacilares da hanseníase. Foi possível avaliar o grau de incapacidade inicial de $36(32,1 \%)$ dos pacientes incluídos. Desses, $25 \%$ apresentaram grau de incapacidade inicial II. Durante o tratamento poliquimioterápico, 21 (18,8\%) pacientes apresentaram algum efeito colateral, sendo o mais comum a anemia relacionada à dapsona. Cinquenta e nove $(52,7 \%)$ pacientes apresentaram algum tipo de estado reacional da hanseníase e 10 (8,9\%) apresentaram complicações sistêmicas graves durante o acompanhamento. Conclusões: O presente estudo evidenciou um perfil clínico-epidemiológico diferente do descrito na literatura, com uma grande porcentagem de pacientes com grau de incapacidade inicial II, uma alta frequência de estados reacionais e de complicações advindas do curso clínico da doença ou dos tratamentos implementados.
\end{abstract}

Palavras-Chave: Hanseníase. Terapêutica. Doenças Negligenciadas.

\begin{abstract}
Introduction: This was an observational, longitudinal, retrospective and descriptive study of a cohort of patients. Objectives: To describe the clinical and epidemiological profile of the patients diagnosed
\end{abstract}

1. Doutorado em Medicina. Professor adjunto de Doenças Infecciosas e Parasitárias da Universidade Federal do Estado do Rio de Janeiro (UNIRIO).

2. Residente em Hansenologia, Especialização em Dermatologia, Hospital Universitário Gaffrée e Guinle, UNIRIO.

3. Especialização em Dermatologia. Professor adjunto de Dermatologia da UNIRIO.

4. Mestranda em HIV/AIDS. Enfermeira da Dermatologia, Hospital Universitário Gaffrée e Guinle, UNIRIO.

5. Mestrado em Neurociências. Professor adjunto de Dermatologia da UNIRIO.
CORRESPONDÊNCIA: Vinicius Martins de Menezes Hospital Universitário Gaffrée e Guinle, Departamento de Medicina Geral, Rua Mariz e Barros, 775 20270-001 - Rio de Janeiro/RJ - Brasil vmenezes70@gmail.com

Recebido em 24/07/2018 Aprovado em 30/01/2019 
with leprosy admitted to a reference medical center in the State of Rio de Janeiro. Methods: All leprosy patients admitted to the dermatology clinic from the Hospital Universitário Gaffrée and Guinle between 2008 and 2017 were included. Pertinent patients' data were collected through medical charts review. Data were then analyzed through descriptive statistics using SPSS 22.0 software. Results: a total of 112 patients were included in the study. Among them, 54.5\% were male, the median age was 49.9 years old, $62.4 \%$ were residents in the city of Rio de Janeiro and $69.9 \%$ presented multibacillary leprosy clinical forms. We evaluated the initial disability grade of 36 from the 112 included patients, and out of them, 25\% had grade II disability. During multidrug therapy, 21 (18.8\%) patients presented an adverse effect, which the most common was anemia related to dapsone. Fiftynine $(52.7 \%)$ patients presented leprosy reaction and $10(8.9 \%)$ patients presented severe systemic complications during the follow-up. Conclusions: In the present study, it was observed a distinctive clinical and epidemiological leprosy profile. There was a high frequency of grade II initial disability, leprosy reaction and severe clinical complications due to the progression of the disease or as adverse effects of leprosy treatment.

Keywords: Leprosy. Therapeutics. Neglected Diseases.

\section{INTRODUÇÃO}

A hanseníase é uma doença crônica e infecciosa causada pelo Mycobacterium leprae, bacilo intracelular obrigatório, que afeta principalmente a pele, as mucosas e os nervos periféricos. ${ }^{1} \mathrm{~A}$ principal forma de transmissão da doença é o convívio com pacientes portadores de formas clínicas multibacilares e sem tratamento, através da via respiratória. ${ }^{1}$

A capacidade de gerar danos permanentes e deformidades está intimamente relacionada à interação entre o sistema imunológico e o patógeno, conferindo amplo espectro clínico à doença. De acordo com a classificação de Madri, a doença pode ser dividida em indeterminada, tuberculoide, virchowiana e dimorfa (borderline). ${ }^{2}$ Alguns pacientes não apresentam lesões visíveis na pele e podem ter lesões apenas nos nervos (hanseníase neural pura). ${ }^{2}$

Com a finalidade de facilitar o diagnóstico e a implementação do tratamento com a poliquimioterapia (PQT) para os níveis primários de saúde, a Organização Mundial da Saúde (OMS) recomenda a classificação operacional para a doença, segundo o número de lesões cutâneas: casos paucibacilares (PB), quando apresentarem até cinco lesões ou casos multibacilares (MB), quando houver mais de cinco lesões e/ou baciloscopia positiva. ${ }^{3} \mathrm{~A}$ baciloscopia de esfregaço cutâneo deve ser utilizada como exame complementar, sempre que disponível. O resultado negativo da baciloscopia não exclui o diagnóstico de hanseníase. ${ }^{3}$
A introdução da PQT recomendada pela OMS foi decisiva para as expressivas mudanças no perfil epidemiológico da doença no mundo, mas sua magnitude e $o$ alto poder incapacitante da hanseníase ainda a colocam como problema de saúde pública em muitos países, inclusive no Brasil.

Segundo a OMS, em 2016 foram reportados 214.783 casos novos de hanseníase, o que representa uma taxa de deteç̧ão de 2,9 casos por 100 mil habitantes. ${ }^{4}$ Nesse mesmo ano, foram notificados, no Brasil, 25.218 casos novos, correspondendo a uma taxa de deteç̧ão de 12,2/100.000 habitantes, classificando o país como de alta carga para a doença e em segundo lugar no mundo em número de casos novos da doença. ${ }^{4}$ Todavia, a distribuição dos casos no país não é homogênea, verificando-se regiões de alta prevalência e incidência como as regiões norte e centro-oeste, contrastando com regiões de baixa carga da doença como as regiões sul e sudeste. No estado do Rio de Janeiro, a taxa de prevalência da hanseníase diminuiu de 4,4/10.000 habitantes em 1998 para 0,5/10.000 habitantes em $2016^{5}$ e a taxa de deteç̧ão de casos novos apresentou uma queda de 23,9/100.000 habitantes em 1998 para 4,3/100.000 habitantes em 2016. ${ }^{6}$

As unidades de atenção básica têm papel crucial na detecção precoce, promoção de ações preventivas e curativas para a doença. Durante o curso da doença, 10 a $50 \%$ dos pacientes podem desenvolver complicações inflamatórias agudas que afetam principalmente a pele e os nervos periféricos, classifica- 
das em reação hansênica tipo 1 (ou reversa) e reação hansênica tipo 2 (eritema nodoso hansênico). ${ }^{7}$ As reações hansênicas refletem o fenômeno de hipersensibilidade aos antígenos do Mycobacterium leprae. Nesses casos de hanseníase complicada, a rede de atendimento de maior complexidade, como os ambulatórios especializados e os hospitais gerais, é importante. Além disso, ao nível da atenção primária, alguns casos podem gerar dúvidas quanto ao diagnóstico, recidivas e reações medicamentosas graves.

Nesse cenário, o Hospital Universitário Gaffrée e Guinle (HUGG) é um dos hospitais terciários da região metropolitana do Rio de Janeiro que recebem os casos de hanseníase complicada do Sistema Nacional de Regulação. Esses casos são avaliados por equipe multidisciplinar especializada e seguem em acompanhamento complementar no serviço. Diante de uma grande demanda para seguimento de pacientes com hanseníase, desde 2017 o hospital conta com vagas para o programa de residência médica em hansenologia. Tal programa constitui o quarto ano de residência para os profissionais formados em dermatologia, com o objetivo de valorizar o cuidado dessa doença negligenciada.

Esse trabalho de pesquisa dos residentes de hansenologia objetiva melhorar a compreensão da doença no Estado do Rio de Janeiro.

\section{MATERIAL E MÉTODO}

Estudo descritivo, longitudinal, observacional e retrospectivo de uma coorte de pacientes em que foram incluídos todos os pacientes com diagnóstico de hanseníase no ambulatório de dermatologia do Hospital Universitário Gaffrée e Guinle (HUGG) entre os anos de 2008 e 2017. Atualmente, o HUGG é um centro de referência para assistência e pesquisa em hanseníase no Rio de Janeiro.

- A classificação da forma clínica da hanseníase foi realizada através dos critérios de Madrid, no início do acompanhamento do paciente.

- Índice baciloscópico (IB): realizado no material de esfregaço dérmico, coletado em 4 sítios (lóbulos auriculares, cotove- los, e/ou lesão cutânea) e corado pela técnica de Zielh Nielsen.

- Grau de incapacidade inicial (GII): determinado pela avaliação neurológica simplificada no momento do diagnóstico da hanseníase, conforme recomendação do Ministério da Saúde do Brasil. ${ }^{3}$

- Diagnóstico dos estados reacionais: estabelecidos por critérios clínicos e/ou histopatológicos.

- Local de moradia: a distribuição dos pacientes por Áreas Programáticas (APs) no município do Rio de Janeiro seguiu o modelo adotado pela Secretaria Municipal de Saúde em que os 160 bairros da cidade são agrupados em 10 APs, conforme descrito a seguir: 1.0 (Centro e adjacências), 2.1 (Zona Sul), 2.2 (Grande Tijuca), 3.1 (região da Leopoldina), 3.2 (Grande Méier), 3.3 (Região de Madureira e adjacências), 4.0 (Região de Jacarepaguá e adjacências), 5.1 (Região de Bangu e adjacências), 5.2 (Região de Campo Grande e adjacências), 5.3 (Região de Santa Cruz e adjacências). ${ }^{8}$

Os dados foram coletados através da revisão de prontuários de cada paciente no HUGG e armazenados em planilha do programa Excel (versão 2010). Posteriormente, os dados foram analisados, utilizando-se o software SPSS 22.0. A descrição das variáveis foi realizada utilizando-se as frequências absoluta e relativa.

Este estudo foi conduzido de acordo com os padrões estabelecidos nas Boas Normas de Práticas Clínicas do Documento das Américas da Organização Pan-Americana de Saúde. 90 arquivamento dos dados de registro dos pacientes foi mantido em sigilo e não houve divulgação de qualquer dado que pudesse identificar o paciente.

\section{RESULTADOS}

Entre 2008 e 2017, 112 pacientes com diagnóstico confirmado de hanseníase foram acompanhados no ambulatório de dermatologia do Hospital Universitário Gaffrée e Guinle e incluídos no presente estudo. A Figura 1 mostra a distribuição dos casos por ano do diagnóstico. 


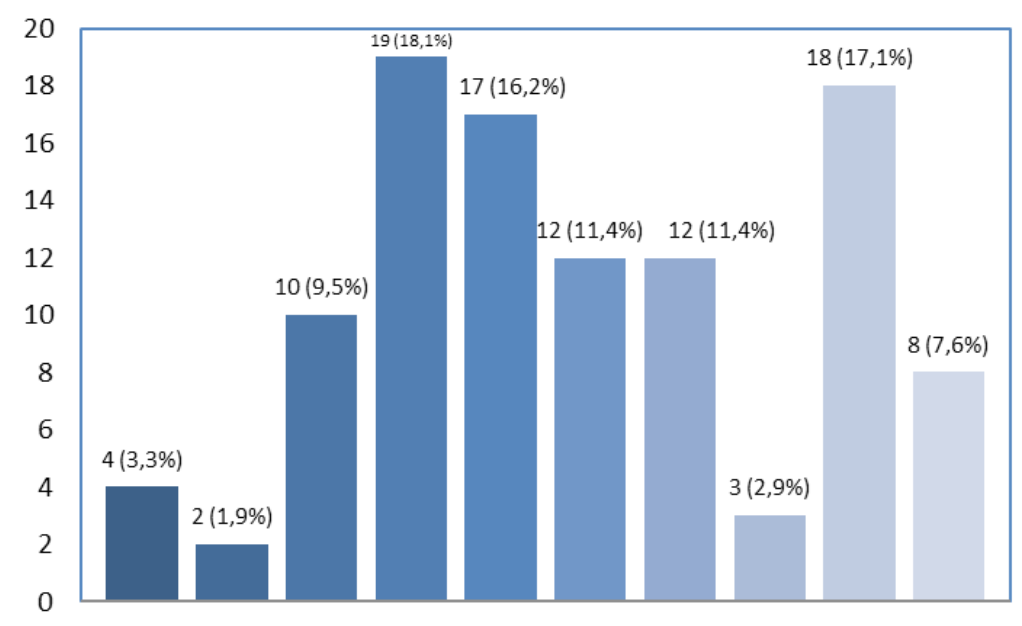

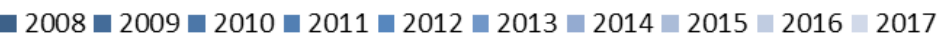

Figura 1: Distribuição dos casos de hanseníase acompanhados no ambulatório de dermatologia do Hospital Universitário Gaffrée e Guinle por ano de diagnóstico.

Dentre os casos incluídos no estudo, 63 Trinta e oito casos $(37,6 \%)$ eram provenientes de $(62,4 \%)$ eram moradores do município do Rio outros municípios, com um maior predomínio de de Janeiro, principalmente das regiões das áre- moradores de São João de Meriti e Belford Roxo as programáticas 2.2, 3.3 e 1.0 (Figura 2). (Figura 2).

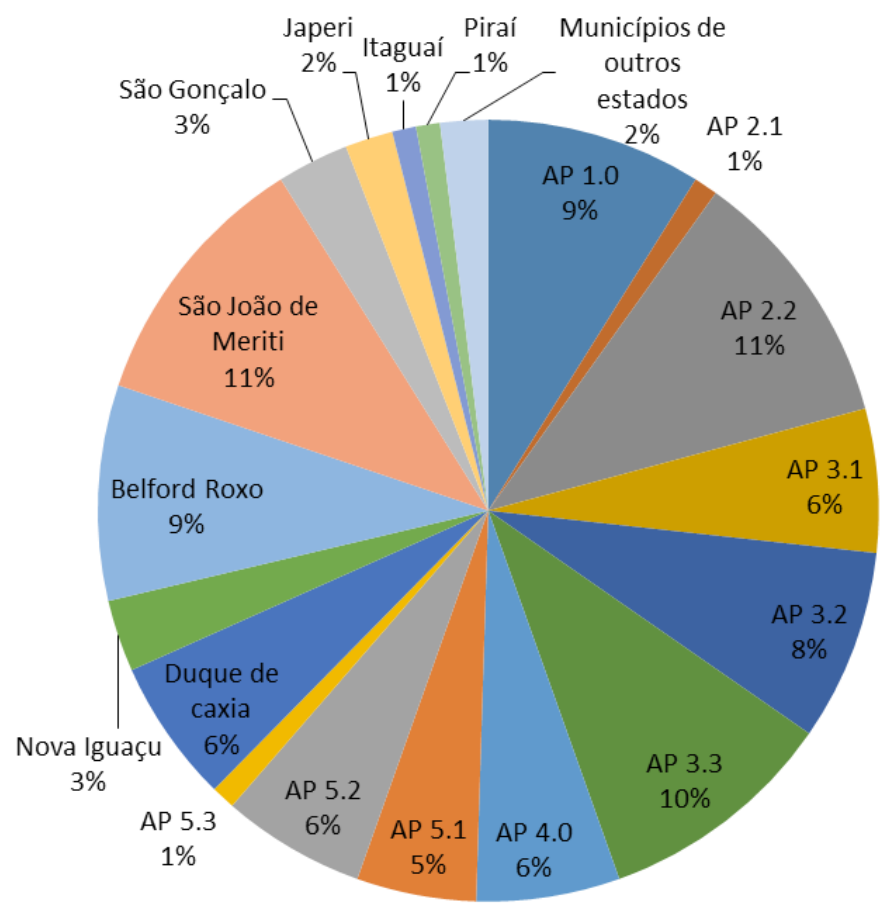

Figura 2: Distribuição dos casos de hanseníase acompanhados no ambulatório de dermatologia do Hospital Universitário Gaffrée e Guinle por local de moradia. AP= Áreas Programáticas. 
Houve uma maior proporção de pacientes do sexo masculino $(54,5 \%)$ e a média de idade foi de 49,9 anos, variando entre 11 e 89 anos (Tabela 1). Três $(2,7 \%)$ dos pacientes incluídos eram menores de 15 anos no momento do diagnóstico da hanseníase. Em relação à classificação clínica da hanseníase, $69,9 \%$ dos pacientes apresentaram formas clínicas multibacilares: dimorfa e Virchowiana (Tabela 1).
Dentre os $57(50,8 \%)$ pacientes em que foi possível a coleta de esfregaço cutâneo no momento do diagnóstico da doença, 17 (29,8\%) apresentavam baciloscopia positiva (Tabela 1). Foi possível avaliar o grau de incapacidade no momento do diagnóstico da hanseníase de $36(32,1 \%)$ pacientes. Desses, 9 $(25 \%)$ apresentavam grau de incapacidade inicial igual a 2 (Tabela 1).

\section{Tabela 1}

Características epidemiológicas e clínicas dos pacientes no momento do diagnóstico de hanseníase.

\begin{tabular}{lc}
\hline Características & Frequência \\
\hline Sexo Masculino & $61(54,5 \%)$ \\
Idade Média & 49,9 anos \\
Forma Clínica Indeterminada & $8(7,8 \%)$ \\
Forma Clínica Neural Pura & $3(2,9 \%)$ \\
Forma Clínica Tuberculoide & $22(17,3 \%)$ \\
Forma Clínica Dimorfa & $40(38,8 \%)$ \\
Forma Clínica Virchowiana & $32(31,1 \%)$ \\
Baciloscopia de esfregaço cutâneo positiva & $17(29,8 \%)$ \\
Grau de incapacidade inicial II & $9(25 \%)$ \\
\hline
\end{tabular}

Setenta e nove $(71,8 \%)$ pacientes foram tratados com o esquema de poliquimioterapia multibacilar. Desses, 4 (5\%) pacientes necessitaram de curso prolongado de 24 meses, $3(3,8 \%)$ foram tratadas sem o uso de dapsona por estarem grávidas e $1(1,3 \%)$ recebeu tratamento para multibacilar com blister infantil (Tabela 2). Trinta e um $(28,2 \%)$ pacientes foram tratados com o esquema poliquimioterápico paucibacilar, sendo que $1(3,2 \%)$ tratou com blister infantil (Tabela 2 ).

Durante o tratamento poliquimioterápico, $21(18,8 \%)$ pacientes apresentaram algum efeito colateral às drogas que compõem o esquema terapêutico e necessitaram de suspensão. (Tabela 2). Desses, 18 (85,6\%) apresentaram anemia ao uso de dapsona, $1(4,7 \%)$ evoluiu com pancitopenia após a introdução da poliquimioterapia, sendo necessária a suspensão temporária do esquema; $1(4,7 \%)$ apresentou hepatite medicamentosa e $1(4,7 \%)$ apresentou reação de hipersensibilidade à poliquimioterapia e foi tratado com esquema de rifampicina, ofloxacina e minociclina.

Durante a evolução clínica da hanseníase, do momento do diagnóstico ao período após o fim do tratamento, $59(52,7 \%)$ pacientes apresen- taram episódios de estado reacional (Tabela 2). Desses, $30(50,8 \%)$ evoluíram com reação do tipo $1,13(22 \%)$ apresentaram reação do tipo 2 e $16(27,1 \%)$ apresentaram episódios tanto de reação do tipo 1 quanto do tipo 2 . Dentre aqueles que apresentaram reação tipo 2 , um $(7,6 \%)$ deles evoluiu com aspecto clínico peculiar semeIhante à Síndrome de Sweet.

Todos os pacientes foram tratados para os respectivos tipos de estados reacionais com drogas recomendadas pelo Ministério da Saúde do Brasil, entretanto $22(37,9 \%)$ casos necessitaram de tratamentos imunossupressores alternativos associados (Tabela 2). Entre esses, 20 (34,5\%) foram tratados também com azatioprina, $4(6,9 \%)$ com metotrexato e $1(1,7 \%)$ com micofenolato.

Durante o seguimento dos pacientes, nove indivíduos (8\%) apresentaram algum tipo de intercorrência clínica. Destes, 3 (33,3\%) evoluíram com mal perfurante plantar, $2(22,2 \%)$ com tromboembolismo pulmonar, $1(1,1 \%)$ com trombose venosa profunda, $1(1,1 \%)$ paciente foi à óbito por infarto agudo do miocárdio e $1(1,1 \%)$ paciente apresentou coinfecção por histoplasmose durante o acompanhamento ambulatorial. 
Tabela 2

Características da evolução clínica da hanseníase

\begin{tabular}{lc}
\hline Características & Frequência \\
\hline Poliquimioterapia para hanseníase multibacilar & $79(71,8 \%)$ \\
Presença de efeito adverso à poliquimioterapia & $21(18,8 \%)$ \\
Frequência dos estados reacionais da hanseníase & $59(52,7 \%)$ \\
Tratamento dos estados reacionais com drogas & $22(37,9 \%)$ \\
alternativas associadas & $09(8 \%)$ \\
Intercorrências clínicas & 09. \\
\hline
\end{tabular}

\section{DISCUSSÃO}

No presente estudo, através da análise da distribuição dos casos por ano de diagnóstico, verificou-se uma grande variação do número de pacientes atendidos em cada ano avaliado. Esse resultado não reflete a real frequência da doença no estado do Rio de Janeiro, uma vez que pelos dados do Sistema de informação de Agravos de Notificação (SINAN), verifica-se uma diminuição linear da incidência e prevalência da doença nos últimos 10 anos. ${ }^{5,6}$ Uma série de fatores pode estar relacionada a essa variação, tais como, facilidades ou dificuldades no fluxo de encaminhamento e variação da quantidade de vagas para consultas ambulatoriais ofertadas pelo HUGG em diferentes períodos.

Pelas limitações do desenho do estudo não foi possível relacionar a distribuição dos pacientes por local de moradia descrita com a total distribuição dos casos notificados de hanseníase no estado do Rio de Janeiro. A localização do HUGG na região da AP 2.2 pode ter influenciado um maior número de encaminhamentos de pacientes provenientes desta área programática e de regiões vizinhas. Entretanto, verifica-se maior número de pacientes da região norte da cidade do Rio de Janeiro e de outros municípios que compõem a região metropolitana da capital do estado. Essas regiões são caracterizadas por uma grande densidade populacional e baixo nível socioeconômico, fatores facilitadores para a transmissão da doença. Destaca-se que o grande número de pacientes provenientes de outros municípios evidencia uma importante função que os hospitais universitários exercem como centros de referências para os municípios com maior limitação nas suas respectivas redes de atenção especializada à saúde.
As características de gênero e idade dos pacientes incluídos no estudo foram similares às observadas entre os casos de hanseníase notificados no Brasil. Os dados do SINAN mostram maior proporção de casos no sexo masculino e a faixa etária mais comumente reportada foi de 30 a 59 anos. ${ }^{4}$ A proporção de $2,7 \%$ de pacientes menores de 15 anos de idade foi inferior à reportada pelo SINAN durante o mesmo período do estudo e também inferior às descritas em outros estudos de série de casos. ${ }^{10,11}$ O Rio de Janeiro é considerado, atualmente, estado de baixa endemicidade de hanseníase, porquanto os casos em crianças tendem a ser mais raros.

Evidenciou-se, nesta pesquisa, uma maior porcentagem de pacientes com diagnóstico da forma clínica multibacilar da hanseníase. Esses resultados são congruentes com os dados reportados pelo SINAN, em que se verificou que $66,4 \%$ dos casos notificados em todo o Brasil apresentavam a forma clínica multibacilar. ${ }^{3}$ Igualmente, em outras pesquisas realizadas em centros de referências e hospitais universitários também se observou maior predomínio de pacientes com a classificação clínica multibacilar. ${ }^{7,11}$ Apesar da rotina da avaliação do grau de incapacidade inicial dos pacientes, apenas $32,1 \%$ dos pacientes tiveram esses dados registrados no prontuário. A alta taxa de grau II de incapacidade física verificada no presente trabalho, $25 \%$ entre os pacientes avaliados, é superior à de outras casuísticas descritas. ${ }^{12,13}$ Estudo prévio realizado em outro hospital universitário também verificou frequência maior de pacientes com grau II de incapacidade física. ${ }^{11}$ Os hospitais universitários e centros de referência para o tratamento de hanseníase costumam receber pacientes com doenças mais graves ou que demandam maior dificuldade no manejo clínico, o que poderia explicar a maior proporção de pacientes multibacilares e a alta taxa de incapacidade grau II. 
O tratamento da hanseníase é ambulatorial, utilizando-se esquemas terapêuticos padronizados, de acordo com a classificação operacional. ${ }^{3}$ Esses medicamentos podem ter efeitos tóxicos, fazendo com que mudanças no regime de tratamento sejam necessárias. ${ }^{14}$ No presente estudo, 21 (18,8\%) pacientes apresentaram alguma reação adversa às drogas que compõem o esquema terapêutico e necessitaram de suspensão da droga; a dapsona foi a medicação que mais frequentemente acarretou efeitos colaterais. Em um estudo realizado na Índia, Nair et al. (2018) observaram a ocorrência de reações adversas aos medicamentos em 3,1\% dos pacientes e a dapsona foi a droga mais comumente envolvida, em $60,7 \%$ dos casos. ${ }^{14}$ Nesse estudo, o efeito colateral mais frequentemente relacionada à dapsona foi a hepatite medicamentosa, seguida da reação exantemática à droga. ${ }^{14}$ No estudo de Guragain et al. (2017), as reações adversas mais frequentes relatadas foram a icterícia, seguida de dermatite esfoliativa e por último a anemia. ${ }^{15}$ No presente trabalho, o efeito colateral mais comumente relacionado à dapsona foi a anemia, observada em $18(85,6 \%)$ pacientes. Na pesquisa de Deps et al. (2007), em que se incluiu somente pacientes brasileiros, a droga que mais comumente provocou reações adversas foi a dapsona (85 pacientes), seguida pela rifampicina (24 pacientes) e clofazimina (18 pacientes). ${ }^{16}$ Os efeitos colaterais mais comuns provocados pela dapsona foram a anemia hemolítica em 48 casos $(56,5 \%)$. As alterações hepáticas ocorreram em 20 casos $(23,5 \%)$ e as reações cutâneas em 6 casos $(7 \%)^{16} \mathrm{Em}$ outro estudo brasileiro, Gonçalves et al. (2012) observaram que a reação adversa mais comum foi a anemia hemolítica causada pela dapsona. ${ }^{17}$ Os resultados encontrados no presente estudo estão de acordo com os descritos em estudos prévios, sugerindo que nos pacientes brasileiros a anemia é o efeito colateral mais comumente relacionado à dapsona. Essa diferença entre os resultados na Ásia e no Brasil pode estar relacionada a fatores raciais. No estudo de Nair et al. (2018), ocorreu apenas 1 caso de reação pseudo-gripal com a rifampicina e não ocorreu nenhuma reação adversa à clofazimina. ${ }^{14} \mathrm{Em}$ nosso estudo não ocorreram reações adversas à rifampicina e à clofazimina, indicando também a baixa incidência de efeitos colaterais a esses medicamentos.

Os estados reacionais da hanseníase constituem um aspecto clínico importante na evolução clínica da hanseníase. Caracterizam-se por episódios agudos ou subagudos, ocorrendo antes, durante ou após a poliquimioterapia e o seu diagnóstico precoce deve ser feito para evitar danos nervosos permanentes. No presente trabalho, 59 $(52,7 \%)$ pacientes apresentaram episódios de estado reacional, sendo que a maioria apresentou reação tipo 1 . A frequência dos estados reacionais é variável na literatura7 e a comparação entre os diferentes estudos torna-se difícil devido às diferenças nos critérios de definição (por exemplo, incluindo ou excluindo neurite isolada), métodos de diagnóstico (por exemplo, clínica e/ou histopatológico), complexidade do atendimento (por exemplo, hospital geral, centro de referência, atenção primária), fatores étnicos e variações geográficas. Entretanto, é possível afirmar que a frequência encontrada no presente trabalho está de acordo com as taxas mais elevadas relatadas na literatura. Um trabalho realizado em dois centros de referência para hanseníase observou que 55,5\% dos pacientes apresentaram pelo menos um estado reacional. ${ }^{18}$ Outro estudo recente em que foram incluídos pacientes do Brasil, Filipinas e Nepal evidenciou uma frequência de reação de 22,1\% (435/1972 pacientes). ${ }^{19} \mathrm{~A}$ alta frequência de estados reacionais encontradas no presente estudo pode estar relacionada ao fato do HUGG ser um centro de referência para os casos mais graves da doença.

No presente estudo, todos os pacientes com estados reacionais foram tratados com as drogas recomendadas pelo Ministério da Saúde do Brasil. Entretanto 22 pacientes $(37,9 \%)$ necessitaram de outras opções terapêuticas devido a refratariedade ao tratamento convencional ou às condições que contraindicaram o seu uso. A associação de drogas para o tratamento das reações com diferentes mecanismos de ação tem demonstrado grande utilidade ao melhorar a resposta clínica com menores doses de corticoterapia e pelo menor tempo de tratamento. ${ }^{20}$ O predomínio do uso da azatioprina em relação à outras drogas de segunda linha pode estar relacionado ao fato ser mais acessível e maior embasamento na literatura. ${ }^{21}$ 
A reação tipo 2 da hanseníase pode ter apresentações atípicas, como eritema multiforme e reação síndrome de Sweet-símile (SS-símile). O diagnóstico clínico dessa variante de reação tipo 2 é dificultado pela ausência das lesões nodulares características do ENH, predominando as lesões em placas edematosas que frequentemente são mal interpretadas como reação tipo 1. A histopatologia é essencial para a diferenciação da síndrome de Sweet clássica ao se observar achados sugestivos de hanseníase, como por exemplo, histiócitos vacuolizados. ${ }^{22}$ No presente estudo, foi observado um caso de reação hansênica SS-símile, indicando que essa manifestação não é tão incomum.

Além das reações adversas aos medicamentos utilizados no esquema de PQT para tratamento da hanseníase, observou-se, nesta pesquisa, 2 pacientes com tromboembolismo pulmonar (TEP) e 1 paciente com trombose venosa profunda (TVP) que podem estar relacionados ao uso de talidomida e corticoesteroides utilizados para tratamento de estados reacionais. A talidomida também é usada para tratamento do mieloma múltiplo, onde o tromboembolismo venoso (TEV) é um efeito colateral já reconhecido, especialmente quando administrada junto com os corticoesteroides sistêmicos. Nesta doença, o TEV com a talidomida é relatado em menos de $5 \%$ dos casos e o risco aumenta de 12 a $17 \%$ quando é combinada a corticoterapia. ${ }^{23}$ Os relatos de casos de TEV e TEP têm aumentado na literatura, inclusive um deles em paciente com sorologia positiva para HIV. ${ }^{24} \mathrm{Em}$ virtude da frequência desse evento, o Ministério da Saúde do Brasil recomenda o uso de ácido acetilsalićlico $100 \mathrm{mg} /$ dia para profilaxia do tromboembolismo quando a associação de talidomida e corticoide for empregada para tratamento do eritema nodoso hansênico. ${ }^{3}$

Entre as intercorrências clínicas graves, verificou-se duas condições importantes relacionadas ao tratamento com corticoterapia prolongada para o estado reacional do tipo 2 , sem relato de casos semelhantes na literatura. Uma delas foi o óbito por infarto do miocárdio, que ocorreu em paciente tabagista, com doença pulmonar obstrutiva crônica, diabetes mellitus e hipertensão arterial sistêmica, que teve seu quadro clínico agravado durante a corticoterapia. A outra complicação foi a histoplasmose pulmonar aguda, que pode estar relacionada à imunossupressão provocada pelo tratamento. A paciente foi submetida ao tratamento antifúngico com cura da doença.

\section{CONCLUSÕES}

O perfil epidemiológico encontrado foi semelhante ao descrito pela Secretaria de Vigilância em Saúde do Ministério da Saúde do Brasil através da análise do SINAN, em relação ao gênero e idade. Entretanto, observamos alta frequência de estados reacionais da hanseníase, alta porcentagem de pacientes com grau de incapacidade II e alta frequência de intercorrências clínicas durante o período de acompanhamento.

Apesar da diminuição da incidência da doença no Brasil, o rápido reconhecimento das complicações sistêmicas originadas da sua evolução ou dos tratamentos realizados é importante para prevenir sequelas permanentes.

Nesse cenário, programas de residência que visem capacitar médicos dermatologistas na área de hansenologia, de forma a permitir maior eficiência no atendimento dos casos de maior complexidade nos hospitais de atendimento secundário e terciário em hospitais universitários são fundamentais.

\section{REFERÊNCIAS}

1. Cruz RCS, Bührer-Sékula S, Penna MLF, Penna GO, TaIhari S. Leprosy: current situation, clinical and laboratory aspects, treatment history and perspective of the uniform multidrug therapy for all patients. An Bras Dermatol. 2017; 92: 761-73.

2. BRASIL. Ministério da Saúde. Secretaria de Vigilância em Saúde. Departamento de Vigilância das Doenças Transmissíveis. Guia prático sobre a hanseníase. Brasília, 2017. Disponível em: http://portalarquivos2.saude. gov.br/images/pdf/2017/novembro/22/Guia-Pratico-de-Hanseniase-WEB.pdf. Acessado em: 08 de dezembro de 2018.

3. BRASIL. Ministério da Saúde. Secretaria de Vigilância em Saúde. Diretrizes para vigilância, atenção e eliminação da Hanseníase como problema de saúde pública: manual técnico-operacional. Brasília, 2016. Disponível em: http://www.saude.pr.gov.br/arquivos/File/DiretrizesdoManuaTcnicoOperacionaldeHansenase.pdf. Acessado em: 11 de abril de 2018. 
4. BRASIL. Ministério da Saúde. Secretaria de vigilância epidemiológica. Boletim Epidemiológico- Hanseníase. Volume 49, No 4, 2018. Disponível em: http://portalarquivos2.saude.gov.br/images/pdf/2018/fevereiro/19/ 2018-004-Hanseniase-publicacao.pdf. Acessado em 05 de abril de 2018.

5. BRASIL. Ministério da Saúde. Secretaria de vigilância epidemiológica. Taxa de prevalência de hanseníase por 10.000 habitantes. Disponívelem: http://portalarquivos2. saude.gov.br/images/pdf/2017/julho/10/Taxa-de-preval--ncia-de-hansen--ase-1990a2016-.pdf . Acessado em 05 de abril de 2018.

6. BRASIL. Ministério da Saúde. Secretaria de vigilância epidemiológica. Taxa de detecção geral de hanseníase por 100.000 habitantes. Disponível em: http://portalarquivos2.saude.gov.br/images/pdf/2017/julho/10/Taxa-de-detec----o-geral-de-hansen--ase-1990a2016-.pdf. Acessado em 05 de abril de 2018.

7. Teixeira MAG, Silveira VM, Franca ER. Características epidemiológicas e clínicas das reações hansênicas em indivíduos paucibacilares e multibacilares, atendidos em dois centros de referência para hanseníase, na Cidade de Recife, Estado de Pernambuco. Rev Soc Bras Med Trop. 2010; 43: 287-92.

8. Soranz D, Pinto LP, Penna GO. Eixos e a Reforma dos Cuidados em Atenção Primária em Saúde (RCAPS) na cidade do Rio de Janeiro, Brasil. Ciênc Saúde Colet. 2016; 21: $1327-38$.

9. Organização Pan-Americana da Saúde. Boas Práticas Clínicas: Documento das Américas, 2005. Disponível em: http://bvsms.saude.gov.br/bvs/publicacoes/boas_ praticas_clinicas_opas.pdf. Acessado em 11 de abril de 2018.

10. BRASIL. Ministério da Saúde. Secretaria de vigilância epidemiológica. Indicadores epidemiológicos e operacionais de hanseníase- Brasil 2001 - 2016. Disponível em: http://portalarquivos2.saude.gov.br/images/pdf/2017/ julho/10/Indicadores-epidemiol--gicos-e-operacionais-de-hansen--ase.\%20Brasil,\%202001-.pdf. Acessado em 05 de abril de 2018.

11. Queirós MI, Alencar CHM, Sena AL, Ramos Junior AN, Monteiro LD, Barbosa JC. Perfil clínico-epidemiológico de pacientes com hanseníase atendidos em hospital universitário no Ceará entre 2007 e 2011. An Bras Dermatol. 2016; 91: 311-7.

12. Romão ER, Mazzoni AM. Perfil epidemiológico da hanseníase no município de Guarulhos, SP. Rev Epidemiol Controle Infecç. 2013; 3:22-7.

13. Cruz Silva MEG, Souza CDF, Silva SPC, Costa FM, Carmo RF. Epidemiological aspects of leprosy in Juazeiro-BA, from 2002 to 2012. An Bras Dermatol. 2015; 90: 799-805.
14. Nair SP. A 19-Year retrospective study of adverse drug reactions to multidrug therapy in leprosy requiring a change in regime. Indian Dermatol Online J. 2018; 9: 33-6.

15. Guragain S, Upadhayay N, Bhattarai BM. Adverse reactions in leprosy patients who underwent dapsone multidrug therapy: a retrospective study. Clin Pharmacol. 2017; 9:73-8.

16. Deps PD, Nasser S, Guerra P, Simon M, Birshner RDC, Rodrigues LC. Adverse effects from multi-drug therapy in leprosy: a Brazilian study. Lepr Rev. 2007; 78: 216-22.

17. Gonçalves HS, Pontes MAA, Buhrer-Sékula S, Cruz R, Almeida PC, Moraes MEA, Penna GO. Brazilian clinical trial of uniform multidrug therapy for leprosy patients - the correlation between clinical disease types and adverse effects. Mem Inst Oswaldo Cruz. 2012; 107(Suppl. I): 74-8.

18. Hungria EM, Oliveira RM, Penna GO, Aderaldo LC, Pontes MAA, Cruz $R$ et al. Can baseline ML Flow test results predict leprosy reactions? An investigation in a cohort of patients enrolled in the uniform multidrug therapy clinical trial for leprosy patients in Brazil. Infect Dis Poverty. 2016; 5:110.

19. Scollard DM, Martelli CMT, Stefani MMA, Maroja MF, Villahermosa L, Pardillo F, et al. Risk Factors for Leprosy Reactions in Three Endemic Countries. Am J Trop Med Hyg. 2015; 92: 108-14.

20. Nery JAC, Sales AM, Illarramendi X, Duppre NC, Jardim MR, Machado AM. Contribuição ao diagnóstico e manejo dos estados reacionais. Uma abordagem pratica. An Bras Dermatol. 2006; 81: 367-75.

21. Lockwood DNJ, Darlong J, Govindharaj P. AZALEP a randomized controlled trial of azathioprine to treat leprosy nerve damage and Type 1 reactions in India: Main findings. PLoS Negl Trop Dis. 2017; 11:e0005348. doi: 10.1371/journal.pntd.0005348.

22. Gunawan H, Yogya $Y$, Hafinah R, Marsella R, Ermawaty D, Suwarsa O. Reactive Perforating Leprosy, Erythema Multiforme-Like Reactions, Sweet's Syndrome-Like Reactions as Atypical Clinical Manifestations of Type 2 Leprosy Reaction. Int J Mycobacteriol. 2018; 7: 97-100.

23. Yamaguchi S, Yamamoto $Y$, Hosokawa A, Hagiwara $K$, Uezato $\mathrm{H}$, Takahashi K. Deep venous thrombosis and pulmonary embolism secondary to co-administration of thalidomide and oral corticosteroid in a patient with leprosy. J Dermatol. 2012; 39: 711-14.

24. Medeiros S, Fernandes C, Martins N, Machado J, Kutzner $\mathrm{H}$, Afonso A et al. Hansen's disease inan HIV patient complicated by deep vein thrombosis: a rare complication of thalidomide therapy. Eur J Dermatol. 2009; 19: 272-3. 